International Journal of Pure and Applied Mathematics

Volume 87 No. 4 2013, 497-512

ISSN: 1311-8080 (printed version); ISSN: 1314-3395 (on-line version)

url: http://www.ijpam.eu

doi: http://dx.doi.org/10.12732/ijpam.v87i4.1

ijpam.eu

\title{
ON A NONDIFFERENTIABLE VECTOR OPTIMIZATION INVOLVING SEMI E-TYPE-I MAPS IN BANACH SPACES
}

\author{
Hehua Jiao \\ College of Mathematics and Computer \\ Yangtze Normal University \\ Fuling, 408100, Chongqing, P.R.CHINA
}

\begin{abstract}
This paper deals with a vector optimization problem with restrictions of inequalities involving nondifferentiable maps in Banach spaces. Some new concepts of $\eta$-E-semidifferentiablity, semi $E$-invexity and semi $E$-type-I maps in Banach spaces are introduced. A necessary optimality condition and a few sufficient optimality conditions are obtained by generalizing alternative theorem of Gordan type and using semi E-type-I maps. Moreover, weak, strong and converse duality results are proved under various types of semi $E$-type-I maps assumptions.
\end{abstract}

AMS Subject Classification: 90C46, 90C48

Key Words: $\eta$-E-semidifferentiablity, semi $E$-invexity, semi $E$-type-I maps, vector optimization, optimality, duality

\section{Introduction}

Convexity plays a key role in optimality and duality of mathematical programming problems. See, e.g., $[1,2]$. Many attempts have been made during the past several decades to weaken convexity hypothesis [3-21]. In this endeavor, Hanson and Mond[6] introduced a new class of functions called type-I function for a scalar optimization problem, which was further generalized to pseudotype-I and quasi-type-I by Rueda and Hanson[7]. Later, various generalized

Received: November 11, 2012

(c) 2013 Academic Publications, Ltd. url: www.acadpubl.eu 
type-I functions have been introduced and optimality and duality have been established involving these functions[8-15].

Recently, Anurag Jayswal[16] introduced new classes of generalized $\alpha$-univex type-I vector-valued functions and obtained several K-T type sufficient optimality conditions and Mond-Weir type duality results for a multiobjective programming problem with inequality constraints. More recently, Suneja et al.[17] defined generalized type-I functions over cones and established sufficient optimality conditions and duality results for a vector minimization problem using Clarkes generalized gradients. Especially, Yu and Liu[18] obtained some sufficient optimality conditions and duality results for a differentiable vector problem with inequality constraint involving the generalized type-I maps in Banach spaces.

On the other hand, Cristian Niculescu[19] studied optimality and duality for a nonlinear fractional multiobjective programming problem under $\eta$ semidifferentiablity and generalized $\rho$-semilocally type-I preinvexity assumptions. Additionally, Luo and Jian[20] presented semi E-preinvex maps in Banach spaces and discussed their properties.

Motivated by work of [18-20], in the present paper, by generalizing $\eta$ semidifferentiablity proposed by Cristian Niculescu[19] to $\eta$ - $E$-semidifferentiablity, we define a nondifferentiable semi $E$-invex map in Banach spaces and thus extend type-I maps presented by $\mathrm{Yu}$ and Liu[18] to semi E-type-I maps. We obtain a necessary optimality condition by extending alternative theorem of Gordan type and some sufficient optimization conditions by using semi E-typeI maps for a nondifferentiable vector optimization problem with restrictions of inequalities. Moreover, we prove weak, strong and converse duality results under various types of semi E-type-I maps assumptions. Our results generalize and improve some results obtained in the literatures on this topic.

\section{Preliminaries and Definitions}

Throughout this paper, let $X, Y$ and $Z_{j}, j \in M=\{1,2, \ldots, m\}$ be real Banach spaces with topological duals $X^{*}, Y^{*}$ and $Z_{j}^{*}$, respectively, $E: X \rightarrow X$ and $\eta: X \times X \rightarrow X$ be two fixed mappings.

Consider the following optimization problem:

$(P) \quad\left\{\begin{array}{l}\min f(x) \\ \text { s.t. }-g(x)=-\left(g_{1}(x), \ldots, g_{m}(x)\right) \in\left(D_{1} \times, \ldots, \times D_{m}\right), \\ x \in K \subset X\end{array}\right.$ 
where $f: X \rightarrow Y$ and $g_{j}: X \rightarrow Z_{j}$ are maps, $K$ and $D_{j}$ are subsets of $X$ and $Z_{j}$. Denote the feasible set of $(P)$ by $F=\left\{x \in K:-g_{j}(x) \in D_{j}, j \in M\right\}$. We assume that the spaces $Y$ and $Z_{j}$ are ordered by cones $C \subset Y, D_{j} \subset Z_{j}$ and these cones are pointed, closed, convex, and with nonempty interior. The dual cone of $C$ is denoted by

$$
C^{*}=\left\{\mu^{*} \in Y^{*}:\left\langle\mu^{*}, x\right\rangle \geq 0, \forall x \in C\right\} .
$$

The cone $C$ induces a partial order $\leq_{C}$ on $Y$ given by:

$$
\begin{array}{ll}
x, y \in Y, & x \leqq_{C} y \Longleftrightarrow y-x \in C ; \\
x, y \in Y, & x \leq_{C} y \Longleftrightarrow y-x \in C \backslash\left\{0_{Y}\right\} ; \\
x, y \in Y, & x<_{C} y \Longleftrightarrow y-x \in \text { int } C .
\end{array}
$$

Similarly, $D_{j}$ induces a partial order on $Z_{j}, j \in M$.

Recall some definitions and results that will be used in the sequel.

Definition 2.1. ([21]) We say that problem $(P)$ satisfies the Slater regularity condition if there exists $\tilde{x} \in F$ such that $g_{j}(\tilde{x})<_{D_{j}} 0, \quad j \in M$.

Definition 2.2. ([18]) We say that $\bar{x} \in F$ is a weakly efficient solution [or, an efficient solution] of problem $(P)$, if there exists no $x \in F$ such that

$$
f(x)<_{C} f(\bar{x}) \quad\left[o r, f(x) \leq_{C} f(\bar{x})\right] .
$$

Definition 2.3. ([20]) A set $K \subset X$ is said to be $E$-invex with respect to $\eta$ if

$$
E(y)+\lambda \eta(E(x), E(y)) \in K, \quad \forall x, y \in K, \lambda \in[0,1] .
$$

Definition 2.4. ([20]) Let $K \subset X$ be an $E$-invex set with respect to $\eta$. A map $f: X \rightarrow Y$ is said to be semi $E$-preinvex on $K$ with respect to $\eta$ if

$$
f(E(y)+\lambda \eta(E(x), E(y))) \leqq_{C} \lambda f(x)+(1-\lambda) f(y), \quad \forall x, y \in K, \lambda \in[0,1] .
$$

Lemma 2.1. ([22]) Let $C \subset Y$ be a convex cone with int $C \neq \varnothing$ and $C^{*}$ the dual cone of $C$. Then, (a) $\forall \mu^{*} \in C^{*} \backslash\left\{0_{Y^{*}}\right\}, \quad x \in$ int $C \Rightarrow\left\langle\mu^{*}, x\right\rangle>0$; (b) $\forall \mu^{*} \in$ int $^{*}, \quad x \in C \backslash\left\{0_{Y}\right\} \Rightarrow\left\langle\mu^{*}, x\right\rangle>0$.

Now, we introduce some new concepts.

Definition 2.5. Let $f: K \rightarrow Y$ be a map, where $K \subset X$ is an E-invex set with respect to $\eta$. We say that $f$ is $\eta$-E-semidifferentiable at $E(\bar{x}) \in K$ if $f^{\prime}(E(\bar{x}) ; \eta(E(x), E(\bar{x})))$ exists for each $x \in K$, where

$$
f^{\prime}(E(\bar{x}) ; \eta(E(x), E(\bar{x})))=\lim _{\lambda \rightarrow 0^{+}} \frac{1}{\lambda}[f(E(\bar{x})+\lambda \eta(E(x), E(\bar{x})))-f(E(\bar{x}))],
$$

(the right derivative at $E(\bar{x})$ along the direction $\eta(E(x), E(\bar{x}))$ ). 
Remark 2.1. If $E$ is an identity map and $Y=R^{n}$, the $\eta$-E-semidifferentiability is the $\eta$-semidiffer -entiability notion[19]. If $\eta(x, \bar{x})=x-\bar{x}, Y=$ $R^{n}$ and $E$ is an identity map, the $\eta$-E-semidifferentia- bility is the semidifferentiability notion. If a function is directionally differentiable, then it is semidifferentiable, but the converse is not true.

Definition 2.6. Let $K \subset X$ be an $E$-invex set with respect to $\eta$. A map $f: X \rightarrow Y$ is called semi $E$-invex at $\bar{x}$ on $K$ with respect to $\eta$, if $f$ is $\eta$-E-semidifferentiable at $\bar{x} \in K$, where $E(\bar{x})=\bar{x}$ and

$$
f(x)-f(\bar{x}) \geqq_{C} f^{\prime}(\bar{x} ; \eta(E(x), \bar{x})), \quad \forall x \in K,
$$

which is equivalent to the following relation:

$$
\left\langle\mu^{*}, f(x)-f(\bar{x})\right\rangle \geq\left(\mu^{*} \circ f\right)^{\prime}(\bar{x} ; \eta(E(x), \bar{x})), \quad \forall x \in K, \mu^{*} \in C^{*},
$$

(see [21, Lemma 2.3]).

Remark 2.2. If a semi $E$-preinvex map $f$ is $\eta$ - $E$-semidifferentiable at $\bar{x}$, where $E(\bar{x})=\bar{x}$, then $f$ is a semi $E$-invex map at $\bar{x}$.

Next, we extend the generalized type-I maps in [18] as follows.

Definition 2.7. $(f, g)$ is called semi $E$-type-I at $\bar{x} \in K$ with respect to $\eta$, if for each $x \in K$, there exist two maps $E$ and $\eta$ such that $E(\bar{x})=\bar{x}$ and for all $\mu^{*} \in C^{*}, v_{j}^{*} \in D_{j}^{*}, j \in M$

$$
\begin{gathered}
\left\langle\mu^{*}, f(x)-f(\bar{x})\right\rangle \geq\left(\mu^{*} \circ f\right)^{\prime}(\bar{x} ; \eta(E(x), \bar{x})) ; \\
-\sum_{j=1}^{m}\left\langle v_{j}^{*}, g_{j}(\bar{x})\right\rangle \geq \sum_{j=1}^{m}\left(v_{j}^{*} \circ g_{j}\right)^{\prime}(\bar{x} ; \eta(E(x), \bar{x})) .
\end{gathered}
$$

Definition 2.8. $(f, g)$ is called quasi semi $E$-type-I at $\bar{x} \in K$ with respect to $\eta$, if for each $x \in K$, there exist two maps $E$ and $\eta$ such that $E(\bar{x})=\bar{x}$ and for all $\mu^{*} \in C^{*}, v_{j}^{*} \in D_{j}^{*}, j \in M$

$$
\begin{gathered}
\left\langle\mu^{*}, f(x)\right\rangle \leq\left\langle\mu^{*}, f(\bar{x})\right\rangle \Rightarrow\left(\mu^{*} \circ f\right)^{\prime}(\bar{x} ; \eta(E(x), \bar{x})) \leq 0 ; \\
-\sum_{j=1}^{m}\left\langle v_{j}^{*}, g_{j}(\bar{x})\right\rangle \leq 0 \Rightarrow \sum_{j=1}^{m}\left(v_{j}^{*} \circ g_{j}\right)^{\prime}(\bar{x} ; \eta(E(x), \bar{x})) \leq 0 .
\end{gathered}
$$

Definition 2.9. $(f, g)$ is called pseudo semi $E$-type-I at $\bar{x} \in K$ with respect to $\eta$, if for each $x \in K$, there exist two maps $E$ and $\eta$ such that $E(\bar{x})=\bar{x}$ and for all $\mu^{*} \in C^{*}, v_{j}^{*} \in D_{j}^{*}, j \in M$

$$
\left(\mu^{*} \circ f\right)^{\prime}(\bar{x} ; \eta(E(x), \bar{x})) \geq 0 \Rightarrow\left\langle\mu^{*}, f(x)\right\rangle \geq\left\langle\mu^{*}, f(\bar{x})\right\rangle ;
$$




$$
\sum_{j=1}^{m}\left(v_{j}^{*} \circ g_{j}\right)^{\prime}(\bar{x} ; \eta(E(x), \bar{x})) \geq 0 \Rightarrow-\sum_{j=1}^{m}\left\langle v_{j}^{*}, g_{j}(\bar{x})\right\rangle \geq 0 .
$$

Definition 2.10. $(f, g)$ is called quasipseudo semi $E$-type-I at $\bar{x} \in K$ with respect to $\eta$, if for each $x \in K$, there exist two maps $E$ and $\eta$ such that $E(\bar{x})=\bar{x}$ and for all $\mu^{*} \in C^{*}, v_{j}^{*} \in D_{j}^{*}, j \in M$

$$
\begin{gathered}
\left\langle\mu^{*}, f(x)\right\rangle \leq\left\langle\mu^{*}, f(\bar{x})\right\rangle \Rightarrow\left(\mu^{*} \circ f\right)^{\prime}(\bar{x} ; \eta(E(x), \bar{x})) \leq 0 ; \\
\sum_{j=1}^{m}\left(v_{j}^{*} \circ g_{j}\right)^{\prime}(\bar{x} ; \eta(E(x), \bar{x})) \geq 0 \Rightarrow-\sum_{j=1}^{m}\left\langle v_{j}^{*}, g_{j}(\bar{x})\right\rangle \geq 0 .
\end{gathered}
$$

If in the above relation, we have

$$
\sum_{j=1}^{m}\left(v_{j}^{*} \circ g_{j}\right)^{\prime}(\bar{x} ; \eta(E(x), \bar{x})) \geq 0 \Rightarrow-\sum_{j=1}^{m}\left\langle v_{j}^{*}, g_{j}(\bar{x})\right\rangle>0 .
$$

Then, we say that $(f, g)$ is quasistrictlypseudo semi $E$-type-I at $\bar{x} \in K$.

Definition 2.11. $(f, g)$ is called pseudoquasi semi $E$-type-I at $\bar{x} \in K$ with respect to $\eta$, if for each $x \in K$, there exist two maps $E$ and $\eta$ such that $E(\bar{x})=\bar{x}$ and for all $\mu^{*} \in C^{*}, v_{j}^{*} \in D_{j}^{*}, j \in M$

$$
\begin{array}{r}
\left(\mu^{*} \circ f\right)^{\prime}(\bar{x} ; \eta(E(x), \bar{x})) \geq 0 \Rightarrow\left\langle\mu^{*}, f(x)\right\rangle \geq\left\langle\mu^{*}, f(\bar{x})\right\rangle ; \\
-\sum_{j=1}^{m}\left\langle v_{j}^{*}, g_{j}(\bar{x})\right\rangle \leq 0 \Rightarrow \sum_{j=1}^{m}\left(v_{j}^{*} \circ g_{j}\right)^{\prime}(\bar{x} ; \eta(E(x), \bar{x})) \leq 0 .
\end{array}
$$

Remark 2.3. If $(f, g)$ is semi $E$-type-I at $\bar{x} \in K$ with respect to $\eta$, then $(f, g)$ is both quasi semi $E$-type-I and pseudo semi $E$-type-I at $\bar{x} \in K$ with respect to $\eta$. If $E$ is an identity map and $m=1$, then the definitions (2.7)-(2.11) reduce to generalized type-I maps defined by Yu and Liu [18].

\section{Optimality Criteria}

In this section, we establish a necessary and a few sufficient optimality conditions for problem $(P)$.

To obtain the necessary optimality condition, we need to prove the following generalized Gordan type alternative theorem. 
Lemma 3.1. Let a map $f: X \rightarrow Y$ be semi E-preinvex on E-invex set $K \subset X$ with respect to $\eta$, if $C \subset Y$ is a convex cone with nonempty interior. Then, either

(a) there exists $x \in K$, such that $-f(x) \in \operatorname{int} C$, or

(b) there exists $p \in C^{*} \backslash\{0\}$, such that $(p \circ f)(K) \subset R_{+}$, where $R_{+}=\{\alpha \in R$ : $\alpha \geq 0\}$.

Proof. We assume that systems (a) and (b) have solutions $x \in K$ and $p \in C^{*} \backslash\{0\}$. Then, from Lemma 2.1, we have that $(p \circ f)(x)<0, x \in K$, which is a contradiction to (b).

Now, we assume that system (a) has no solution. We will prove that system (b) has a solution.

We put $A=f(K)+\operatorname{int} C$. Then, set $A$ is open. In fact, let $u \in A$, there exists $x \in K$ and $s \in \operatorname{int} C$, such that $u=f(x)+s$. Since $s \in \operatorname{int} C$, there exists a ball $N$ with center at zero, such that $s+N \subset C$. However, $u+N=$ $f(x)+(s+N) \subset A$, and consequently, $A$ is open.

Next, we will prove that $A$ is a convex set. Let $u_{1}, u_{2} \in A$, and $\tau \in(0,1)$. Then, $u_{1}=f\left(x_{1}\right)+s_{1}, u_{2}=f\left(x_{2}\right)+s_{2}$, with $x_{1}, x_{2} \in K$ and $s_{1}, s_{2} \in \operatorname{int} C$.

$$
(1-\tau) u_{1}+\tau u_{2}=\left[(1-\tau) f\left(x_{1}\right)+\tau f\left(x_{2}\right)\right]+\left[(1-\tau) s_{1}+\tau s_{2}\right] .
$$

Since $f$ is semi $E$-preinvex map, we have

$f\left[E\left(x_{1}\right)+\tau \eta\left(E\left(x_{2}\right), E\left(x_{1}\right)\right)\right] \leqq C(1-\tau) f\left(x_{1}\right)+\tau f\left(x_{2}\right), \quad \forall \tau \in(0,1), x_{1}, x_{2} \in K$, namely,

$$
(1-\tau) f\left(x_{1}\right)+\tau f\left(x_{2}\right) \in f\left[E\left(x_{1}\right)+\tau \eta\left(E\left(x_{2}\right), E\left(x_{1}\right)\right)\right]+C,
$$

and

$$
(1-\tau) s_{1}+\tau s_{2} \in \operatorname{int} C .
$$

By hypothesis, $K$ is an $E$-invex set, that is,

$$
E\left(x_{1}\right)+\tau \eta\left(E\left(x_{2}\right), E\left(x_{1}\right)\right) \in K, \quad \forall \tau \in(0,1), x_{1}, x_{2} \in K
$$

From relations (3.1)-(3.4), we obtain $(1-\tau) u_{1}+\tau u_{2} \in A$, i.e., the set $A$ is convex.

Since system (a) has no solution, then $0 \notin A$. From Hahn-Banach theorem, there exists $p \in Y^{*} \backslash\{0\}$, such that

$$
p(A) \subset R_{+}
$$


We fix $s \in$ intC. We would like to prove: $p(f(x)) \geq 0, \forall x \in K$. Since $s \in$ int $C$, we have

$$
s+N \subset \text { int } C, \quad \text { for some ball } N \text {. }
$$

For $\tau \in R_{+}$sufficiently big, we have $\frac{1}{\tau} f(x) \in N$ and from (3.6) we have $s-$ $\frac{1}{\tau} f(x) \in \operatorname{int} C$ and considering that intC is also a cone, we obtain $\tau s-f(x) \in$ int $C$, that is $\tau s \in f(x)+i n t C \subset A$, and therefore, by (3.5) we have

$$
p(s) \geq 0, \quad \forall s \in \text { int } C .
$$

However, for each $\varepsilon>0$ sufficiently small, such that $u=f(x)+\varepsilon s \in A$, and therefore,

$$
(p \circ f)(x)=p(u)-\varepsilon P(s) \geq-\varepsilon P(s) \rightarrow 0, \quad \text { as } \varepsilon \rightarrow 0^{+},
$$

consequently,

$$
(p \circ f)(x) \geq 0, \quad \forall x \in K .
$$

For each $s_{0} \in C, p\left(s_{0}\right)=\frac{1}{\tau} p\left(\tau s_{0}\right)$ and for $\tau>0$ small, $\tau s_{0} \in$ int $C$, therefore, by (3.7), we have $p\left(s_{0}\right) \geq 0, \forall s_{0} \in \operatorname{int} C$, that is,

$$
p \in C^{*} \backslash\{0\} .
$$

Thus, (3.8) and (3.9) imply that $p$ is a solution of system (b).

The proof is completed.

Theorem 3.1. (Necessary Optimality) Suppose that $f$ and $g_{j}, j \in M$ are semi $E$-preinvex maps on $E$-invex set $K \subset X$ with respect to $\eta$ and all $\eta$-E-semidifferentiable at $\bar{x} \in K$, where $E(\bar{x})=\bar{x}$. If $\bar{x}$ is a weakly efficient solution of $(P)$, then there exist $\mu^{*} \in C^{*}, v_{j}^{*} \in D_{j}^{*}$, not all zero, such that

$$
\left(\mu^{*} \circ f\right)^{\prime}(\bar{x} ; \eta(E(x), \bar{x}))+\sum_{j=1}^{m}\left(v_{j}^{*} \circ g_{j}\right)^{\prime}(\bar{x} ; \eta(E(x), \bar{x})) \geq 0, \quad \forall x \in F,
$$

and

$$
\sum_{j=1}^{m}\left\langle v_{j}^{*}, g_{j}(\bar{x})\right\rangle=0
$$

Proof. From Proposition 3 in [20], it follows that the feasible set $F=\{x \in$ $\left.K:-g_{j}(x) \in D_{j}, j \in M\right\}$ is $E$-invex set with respect to $\eta$. Let $\bar{x}$ be a weakly efficient solution of $(P)$. In this case, the system

$$
-\left[(f(x)-f(\bar{x})) \times g_{j}(x)\right] \in \operatorname{int}\left(C \times D_{j}\right), \quad j \in M,
$$


has no solution $x \in F$.

From Lemma 3.1, there exists $p=\left(\tau^{*}, v_{j}^{*}\right) \in\left(C^{*}, D_{j}^{*}\right) \backslash\{0,0\}$, such that

$$
\tau^{*} \circ[f(x)-f(\bar{x})]+v_{j}^{*} \circ g_{j}(x) \geq 0, \quad j \in M, \quad x \in F,
$$

consequently,

$$
v_{j}^{*} \circ g_{j}(\bar{x}) \geq 0, \quad j \in M
$$

Also, $\bar{x} \in F$ implies

$$
v_{j}^{*} \circ g_{j}(\bar{x}) \leq 0, \quad j \in M .
$$

Thus, from the above two relations, it follows that

$$
v_{j}^{*} \circ g_{j}(\bar{x})=0, \quad j \in M .
$$

Since $F$ is $E$-invex set and $f, g_{j}, j \in M$ are $\eta$-E-semidifferentiable at $\bar{x}$, where $E(\bar{x})=\bar{x}$, from $(3.12)$ and (3.13), we obtain

$$
\begin{aligned}
& \lim _{\lambda \rightarrow 0^{+}} \frac{\tau^{*} \circ f(\bar{x}+\lambda \eta(E(x), \bar{x}))-\tau^{*} \circ f(\bar{x})+v_{j}^{*} \circ g_{j}(\bar{x}+\lambda \eta(E(x), \bar{x}))-v_{j}^{*} \circ g_{j}(\bar{x})}{\lambda} \\
& =\left(\tau^{*} \circ f\right)^{\prime}(\bar{x} ; \eta(E(x), \bar{x}))+\left(v_{j}^{*} \circ g_{j}\right)^{\prime}(\bar{x} ; \eta(E(x), \bar{x})) \geq 0, \quad j \in M, \quad x \in F .
\end{aligned}
$$

Hence, from (3.13) and (3.14), respectively, it follows

$$
\sum_{j=1}^{m}\left\langle v_{j}^{*}, g_{j}(\bar{x})\right\rangle=0
$$

and

$$
m\left(\tau^{*} \circ f\right)^{\prime}(\bar{x} ; \eta(E(x), \bar{x}))+\sum_{j=1}^{m}\left(v_{j}^{*} \circ g_{j}\right)^{\prime}(\bar{x} ; \eta(E(x), \bar{x})) \geq 0, \quad x \in F .
$$

Setting in (3.15) $m \tau^{*}=\mu^{*}$, we obtain the desirable result.

The following corollary follows directly from Theorem 3.1 and the proof is omitted here.

Corollary 3.1. On the hypotheses of Theorem 3.1, if the Slater regularity condition is verified, then $\mu^{*} \neq 0$.

Now, we establish some sufficient optimality conditions for $(P)$ using semi E-type- $I$ maps. 
Theorem 3.2. Assume that there exist $\bar{x} \in F$ and $\mu^{*} \in C^{*} \backslash\left\{0_{Y^{*}}\right\}$ [or, $\left.\mu^{*} \in i n t C^{*}\right], v_{j}^{*} \in D_{j}^{*}, j \in M$ such that (3.10) and (3.11) hold. Furthermore, if any one of the following conditions holds:

(a) $(f, g)$ is semi $E$-type-I at $\bar{x} \in F$ with respect to the same $\eta$;

(b) $(f, g)$ is pseudoquasi semi E-type-I at $\bar{x} \in F$ with respect to the same $\eta$;

(c) $(f, g)$ is quasistrictlypseudo semi E-type-I at $\bar{x} \in F$ with respect to the same $\eta$.

Then $\bar{x}$ is a weakly efficient solution [or, an efficient solution] of $(P)$.

Proof. By contradiction, we assume that $\bar{x}$ is not a weakly efficient solution [or, an efficient solution] of $(P)$. Then there is a feasible solution $\breve{x}$ of problem $(P)$ such that

$$
f(\breve{x})<_{C} f(\bar{x}) \quad\left[o r, f(\breve{x}) \leq_{C} f(\bar{x})\right] .
$$

From $\mu^{*} \in C^{*} \backslash\left\{0_{Y^{*}}\right\}$ [or, $\left.\mu^{*} \in i n t C^{*}\right]$ and Lemma 2.1, we have

$$
\left\langle\mu^{*}, f(\breve{x})-f(\bar{x})\right\rangle<0 .
$$

By condition (a), we get

$$
\left(\mu^{*} \circ f\right)^{\prime}(\bar{x} ; \eta(E(\breve{x}), \bar{x}))<0 .
$$

According to relation (3.11) and condition (a), we also obtain

$$
\sum_{j=1}^{m}\left(v_{j}^{*} \circ g_{j}\right)^{\prime}(\bar{x} ; \eta(E(\breve{x}), \bar{x})) \leq 0 .
$$

Adding (3.17) and (3.18), we have

$$
\left(\mu^{*} \circ f\right)^{\prime}(\bar{x} ; \eta(E(\breve{x}), \bar{x}))+\sum_{j=1}^{m}\left(v_{j}^{*} \circ g_{j}\right)^{\prime}(\bar{x} ; \eta(E(\breve{x}), \bar{x}))<0,
$$

which is in contradiction with (3.10).

By condition (b) and relation (3.11), we get

$$
\sum_{j=1}^{m}\left(v_{j}^{*} \circ g_{j}\right)^{\prime}(\bar{x} ; \eta(E(\breve{x}), \bar{x})) \leq 0 .
$$

Considering (3.10), we also get

$$
\left(\mu^{*} \circ f\right)^{\prime}(\bar{x} ; \eta(E(\breve{x}), \bar{x})) \geq 0 .
$$


By condition (b) again, we have

$$
\left\langle\mu^{*}, f(\breve{x})-f(\bar{x})\right\rangle \geq 0,
$$

which is a contradiction to (3.16).

By condition (c) and relation (3.16), we obtain

$$
\left(\mu^{*} \circ f\right)^{\prime}(\bar{x} ; \eta(E(\breve{x}), \bar{x})) \leq 0 .
$$

Combining the above inequality with (3.10), we get

$$
\sum_{j=1}^{m}\left(v_{j}^{*} \circ g_{j}\right)^{\prime}(\bar{x} ; \eta(E(\breve{x}), \bar{x})) \geq 0 .
$$

From condition (c) again, it leads to

$$
-\sum_{j=1}^{m}\left\langle v_{j}^{*}, g_{j}(\bar{x})\right\rangle>0,
$$

which contradicts (3.11).

Therefore, the theorem is proved.

\section{Duality}

Consider the following dual for problem $(P)$ :

$(D)\left\{\begin{array}{l}\max \quad f(y) \\ \text { s.t. } \quad\left(\mu^{*} \circ f\right)^{\prime}(y ; \eta(E(x), y))+\sum_{j=1}^{m}\left(v_{j}^{*} \circ g_{j}\right)^{\prime}(y ; \eta(E(x), y)) \geq 0, \\ \forall x \in F \\ \sum_{j=1}^{m}\left\langle v_{j}^{*}, g_{j}(y)\right\rangle \geq 0, \\ y \in K, \quad \mu^{*} \in C^{*}, \quad v_{j}^{*} \in D_{j}^{*}, \quad j \in M .\end{array}\right.$

Denote the feasible set of problem $(D)$ by $G$, i.e., $G=\left\{\left(y, \mu^{*}, v_{j}^{*}\right):\left(\mu^{*} \circ\right.\right.$ $f)^{\prime}(y ; \eta(E(x), y))+\sum_{j=1}^{m}\left(v_{j}^{*} \circ g_{j}\right)^{\prime}(y ; \eta(E(x), y)) \geq 0, \quad \sum_{j=1}^{m}\left\langle v_{j}^{*}, g_{j}(y)\right\rangle \geq$ $\left.0, \quad \forall x \in F, y \in K, \quad \mu^{*} \in C^{*}, \quad v_{j}^{*} \in D_{j}^{*}, \quad j \in M\right\}$.

In this section, we establish weak, strong and converse duality results.

Theorem 4.1. (Weak duality) Let $x \in F,\left(y, \mu^{*}, v_{j}^{*}\right) \in G$, and $\mu^{*} \in$ $C^{*} \backslash\left\{0_{Y^{*}}\right\}$ [or, $\mu^{*} \in$ int $\left.C^{*}\right]$. Furthermore, if any one of the following conditions is satisfied: 
(a) $(f, g)$ is semi E-type- $I$ at $y \in F$ with respect to the same $\eta$;

(b) $(f, g)$ is pseudoquasi semi E-type- $I$ at $y \in F$ with respect to the same $\eta$;

(c) $(f, g)$ is quasistrictlypseudo semi E-type- $I$ at $y \in F$ with respect to the same $\eta$.

Then, $f(x) \nless_{C} f(y)$ [or, $\left.f(x) \not \bigsqcup_{C} f(y)\right]$.

Proof. Assume to the contrary that there exist $\breve{x} \in F, \quad\left(y, \mu^{*}, v_{j}^{*}\right) \in G$ such that

$$
f(\breve{x})<_{C} f(y) \quad\left[\text { or, } f(\breve{x}) \leq_{C} f(y)\right] .
$$

By $\mu^{*} \in C^{*} \backslash\left\{0_{Y^{*}}\right\}$ [or, $\left.\mu^{*} \in \operatorname{int} C^{*}\right]$ and Lemma 2.1, we have

$$
\left\langle\mu^{*}, f(\breve{x})-f(y)\right\rangle<0 .
$$

From $\left(y, \mu^{*}, v_{j}^{*}\right) \in G$, it follows that

$$
-\sum_{j=1}^{m}\left\langle v_{j}^{*}, g_{j}(y)\right\rangle \leq 0
$$

According to the first inequality in (4.1) and $\breve{x} \in F$, we get

$$
\left(\mu^{*} \circ f\right)^{\prime}(y ; \eta(E(\breve{x}), y))+\sum_{j=1}^{m}\left(v_{j}^{*} \circ g_{j}\right)^{\prime}(y ; \eta(E(\breve{x}), y)) \geq 0 .
$$

Utilizing relations (4.2), (4.3) and condition (a), we obtain

$$
\begin{gathered}
\left(\mu^{*} \circ f\right)^{\prime}(y ; \eta(E(\breve{x}), y))<0, \\
\sum_{j=1}^{m}\left(v_{j}^{*} \circ g_{j}\right)^{\prime}(y ; \eta(E(\breve{x}), y)) \leq 0 .
\end{gathered}
$$

Summing the above two inequalities, we have

$$
\left(\mu^{*} \circ f\right)^{\prime}(y ; \eta(E(\breve{x}), y))+\sum_{j=1}^{m}\left(v_{j}^{*} \circ g_{j}\right)^{\prime}(y ; \eta(E(\breve{x}), y))<0
$$

which is a contradiction to relation (4.4).

If condition (b) holds, then $-\sum_{j=1}^{m}\left\langle v_{j}^{*}, g_{j}(y)\right\rangle \leq 0$ implies that

$$
\sum_{j=1}^{m}\left(v_{j}^{*} \circ g_{j}\right)^{\prime}(y ; \eta(E(\breve{x}), y)) \leq 0
$$


Taking (4.4) into account, we obtain

$$
\left(\mu^{*} \circ f\right)^{\prime}(y ; \eta(E(\breve{x}), y)) \geq 0 .
$$

By condition (b) again, the above relation means that

$$
\left\langle\mu^{*}, f(\breve{x})-f(y)\right\rangle \geq 0,
$$

which contradicts (4.2).

If condition (C) holds, then (4.2) leads to

$$
\left(\mu^{*} \circ f\right)^{\prime}(y ; \eta(E(\breve{x}), y)) \leq 0 .
$$

On account of (4.4), we have

$$
\sum_{j=1}^{m}\left(v_{j}^{*} \circ g_{j}\right)^{\prime}(y ; \eta(E(\breve{x}), y)) \geq 0 .
$$

Using condition (C) again, we get

$$
-\sum_{j=1}^{m}\left\langle v_{j}^{*}, g_{j}(y)\right\rangle>0
$$

which is in contradiction with (4.3).

Therefore, the theorem is proved.

Theorem 4.2. (Strong duality) Let $\bar{x}$ be a weakly efficient solution of $(P)$. Assume that the maps $f$ and $g_{j}, j \in M$ are semi $E$-preinvex with respect to the same $\eta$ on $E$-invex set $K \subset X$, are $\eta$-E-semidifferentiable at $\bar{x} \subset K$, where $E(\bar{x})=\bar{x}$, and problem $(P)$ satisfies the Slater regularity condition. Moreover, if any one of the following conditions holds:

(a) $(f, g)$ is semi E-type- $I$ at $x \in F$ with respect to $\eta$;

(b) $(f, g)$ is pseudoquasi semi E-type- $I$ at $x \in F$ with respect to $\eta$;

(c) $(f, g)$ is quasistrictlypseudo semi E-type- $I$ at $x \in F$ with respect to $\eta$.

Then, there exist $\left(\bar{\mu}^{*}, \bar{v}_{j}^{*}\right) \in C^{*} \times D_{j}^{*}$ with $\bar{\mu}^{*} \neq 0_{Y^{*}}$ such that $\sum_{j=1}^{m}\left\langle\bar{v}_{j}^{*}, g_{j}(\bar{x})\right\rangle=$ $0,\left(\bar{x}, \bar{\mu}^{*}, \bar{v}_{j}^{*}\right)$ is a weakly efficient solution for $(D)$, and the objective values of the two problems are equal.

Proof. Since $\bar{x}$ satisfies all the conditions of Theorem 3.1, there exist $\bar{\mu}^{*}, \bar{v}_{j}^{*}$, $j \in M$ such that $\sum_{j=1}^{m}\left\langle\bar{v}_{j}^{*}, g_{j}(\bar{x})\right\rangle=0$ and $\left(\bar{x}, \bar{\mu}^{*}, \bar{v}_{j}^{*}\right) \in G$. Also, by the weak duality, it follows that $\left(\bar{x}, \bar{\mu}^{*}, \bar{v}_{j}^{*}\right)$ is a weakly efficient solution for $(D)$. It is obvious that the objective function values of $(P)$ and $(D)$ are equal at their respective weakly efficient solutions. 
Theorem 4.3. (Converse duality) Let $\left(\bar{y}, \bar{\mu}^{*}, \bar{v}_{j}^{*}\right), j \in M$ be a weakly efficient solution [or, an efficient solution] for problem $(D)$. Assume that $\bar{\mu}^{*} \in$ $C^{*} \backslash\left\{0_{Y^{*}}\right\}$ [or, $\left.\bar{\mu}^{*} \in i n t C^{*}\right]$ and all conditions in Theorem 4.1 hold at $\bar{y}$. Then $\bar{y}$ is a weakly efficient solution [or, an efficient solution] for $(P)$.

Proof. We proceed by contradicting. Assume that $\bar{y}$ is not a weakly efficient solution [or, an efficient solution] for $(P)$, that is, there exists $\breve{y} \in F$ such that

$$
f(\breve{y})<_{C} f(\bar{y}) \quad\left[o r, f(\breve{y}) \leq_{C} f(\bar{y})\right] .
$$

From $\bar{\mu}^{*} \in C^{*} \backslash\left\{0_{Y^{*}}\right\}$ [or, $\left.\bar{\mu}^{*} \in \operatorname{int} C^{*}\right]$ and Lemma 2.1, we have

$$
\left\langle\bar{\mu}^{*}, f(\breve{y})-f(\bar{y})\right\rangle<0 .
$$

By $\left(\bar{y}, \bar{\mu}^{*}, \bar{v}_{j}^{*}\right) \in G, j \in M$, we also have

$$
\left(\bar{\mu}^{*} \circ f\right)^{\prime}(\bar{y} ; \eta(E(\breve{y}), \bar{y}))+\sum_{j=1}^{m}\left(\bar{v}_{j}^{*} \circ g_{j}\right)^{\prime}(\bar{y} ; \eta(E(\breve{y}), \bar{y})) \geq 0,
$$

and

$$
\sum_{j=1}^{m}\left\langle\bar{v}_{j}^{*}, g_{j}(\bar{y})\right\rangle \geq 0
$$

On account of condition (a) in Theorem 4.1 and (4.5), we get

$$
\left(\bar{\mu}^{*} \circ f\right)^{\prime}(\bar{y} ; \eta(E(\breve{y}), \bar{y}))<0 .
$$

Since condition (a) of Theorem 4.1 holds and $\breve{y} \in F, \quad\left(\bar{y}, \bar{\mu}^{*}, \bar{v}_{j}^{*}\right) \in G, j \in M$, it yields that

$$
\sum_{j=1}^{m}\left(\bar{v}_{j}^{*} \circ g_{j}(\bar{y})\right)^{\prime}(\bar{y} ; \eta(E(\breve{y}), \bar{y})) \leq 0 .
$$

From relations (4.8) and (4.9), it follows that

$$
\left(\bar{\mu}^{*} \circ f\right)^{\prime}(\bar{y} ; \eta(E(\breve{y}), \bar{y}))+\sum_{j=1}^{m}\left(\bar{v}_{j}^{*} \circ g_{j}(\bar{y})\right)^{\prime}(\bar{y} ; \eta(E(\breve{y}), \bar{y}))<0
$$

which is a contradiction to (4.6).

If condition (b) or (c) of Theorem 4.1 holds, by the similar argument to that of Theorem 4.1, we obtain

$$
\left\langle\bar{\mu}^{*}, f(\breve{y})-f(\bar{y})\right\rangle \geq 0,
$$


or

$$
-\sum_{j=1}^{m}\left\langle\bar{v}_{j}^{*}, g_{j}(\bar{y})\right\rangle>0 .
$$

The inequalities (4.10) and (4.11) contradict (4.5) and (4.7), respectively.

So the theorem is proved.

\section{Acknowledgments}

This work is supported by National Science Foundation of China (No. 61373174) and supported by Education Committee Research Foundation of Chongqing (No. KJ131314).

\section{References}

[1] O.L. Mangasarian, Nonlinear Programming, McGrawHill, New York (1969).

[2] M.S. Bazaraa, H.D. Sherali, C.M. Shetty, Nonlinear Programming: Theory and Algorithms, Third Ed., Wiley, New York (2006).

[3] R.N. Mukherjee, S.K. Mishra, Multiobjective programming with semilocally convex functions, J. Math. Anal. Appl., 199 (1996), 409-424.

[4] S.K. Suneja, C.S. Lalitha, S. Khurana, Optimality and duality theorems for nonsmooth multiobjective fractional programs, Indian J. Pure Appl. Math., 30 (1999), 243-257.

[5] C. Fulga, V. Preda, Nonlinear programming with E-preinvex and local E-preinvex functions, Eur. J. Oper. Res., 192 (2009), 737-743.

[6] M.A. Hanson, B. Mond, Necessary and sufficient conditions in constrained optimization, Math. Program., 37 (1987), 51-58.

[7] N.G. Rueda, M.A. Hanson, Optimality criteria in mathematical programming involving generalized invexity, J. Math. Anal. Appl., 130 (1988), $375-385$.

[8] F. Zhao, On sufficiency of Kuhn-Tucker conditions in non differentiable programming, Bull. Aust. Math. Soc., 46 (1992), 385-389. 
[9] R.N. Kaul, S.K. Suneja, M.K. Srivastava, Optimality criteria and duality in multiple objective optimization involving generalized invexity, J. Optim. Theory Appl., 80 (1994), 465-482.

[10] H. Kuk, T. Tanino, Optimality and duality in nonsmooth multiobjecive optimization involving generalized type-I functions, Comput. Math. Appl., 45 (2003), 1497-1506.

[11] M. Hachimi, B. Aghezzaf, Sufficiency and duality in nondifferentiable multiobjective programming involving generalized type I functions, J. Math. Anal. Appl., 319 (2006), 110-123.

[12] S.K. Mishra, M.A. Noor, Some nondifferentiable multiobjective programming problems, J. Math. Anal. Appl., 316 (2006), 472-482.

[13] M. Hachimi, B. Aghezzaf, Sufficiency and duality in nondifferentiable multiobjective programming involving generalized type I functions, J. Math. Anal. Appl., 319 (2006), 110-123.

[14] T. Antczak, Optimality conditions and duality for nondifferentiable multiobjective programming problems involving d-r-type I functions, J. Comput. Appl. Math., 225 (2009), 236-250.

[15] H. Slimani, M.S. Radjef, Nondifferentiable multiobjective programming under generalized d $\mathrm{d}_{I}$-invexity, Eur. J. Oper. Res., 202 (2010), 32-41.

[16] A. Jayswal, On sufficiency and duality in multiobjective programming problem under generalized $\alpha$-type I univexity, J. Glob. Optim., 46 (2010), 207-216.

[17] S.K. Suneja, S. Khurana, M. Bhatia, Optimality and duality in vector optimization involving generalized type I functions over cones, J. Glob. Optim., 49 (2011), 23-35.

[18] G.L. Yu, S.Y. Liu, Some vector optimization problems in Banach spaces with generalized convexity, Comput. Math. Appl., 54 (2007), 1403-1410.

[19] C. Niculescu, Optimality and duality in multiobjective fractional programming involving $\rho$-semilocally type I-preinvex and related functions, J. Math. Anal. Appl., 335 (2007), 7-19.

[20] Z.M. Luo, J.B. Jian, Some properties of semi-E-preinvex maps in Banach spaces, Nonlinear Anal., 12 (2011), 1243-1249. 
[21] L. Batista dos Santos, R. Osuna-Gómez, M.A. Rojas-Medar, A. RufiánLizana, Preinvex functions and weak efficient solutions for some vectorial optimization problem in Banach spaces, Comput. Math. Appl., 48 (2004), 885-895.

[22] B.D. Craven, Control and Optimization, Chapman and Hall, London (1995). 